\title{
Esophageal Perforation after Using a Single-Use Disposable Duodenoscope
}

\author{
Bakht S. Cheema ${ }^{a} \quad$ Maged Ghali ${ }^{a}$ Ron Schey ${ }^{a}$ Ziad Awad $^{b}$ \\ Bruno Ribeiro ${ }^{a}$ \\ aDivision of Gastroenterology, Department of Internal Medicine, University of Florida

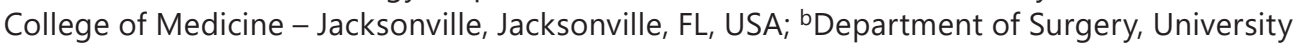 \\ of Florida College of Medicine - Jacksonville, Jacksonville, FL, USA
}

\section{Keywords}

Biliary endoscopy · Diagnostic and therapeutic endoscopy · Endoscopic retrograde cholangiopancreatography · Disposable endoscopes · Duodenoscope reprocessing ·

Perforation · Complications

\begin{abstract}
The Food and Drug Administration (FDA) has recently released a safety communication recommending transition to duodenoscopes with innovative designs that facilitate or eliminate the need for reprocessing. Thus, there has been a significant amount of development into disposable duodenoscope components and single-use duodenoscopes, with variable tactile feedback. We describe a case of esophageal perforation after using a single-use disposable duodenoscope (EXALT Model D; Boston Scientific Corporation, Marlborough, MA, USA). To our knowledge, this is the first reported case of an esophageal perforation since FDA approval of this device in December 2019. ERCP was performed with the EXALT Model D single-use duodenoscope (Boston Scientific Corporation) by an experienced gastroenterologist. During the procedure, gentle force applied through the gastroesophageal junction caused a liner perforation in the distal esophagus. An esophageal stent was placed with satisfactory wound healing and no fistula formation. There have been a few reports in the last 2 years showing promising results using this device; however, the differences in the tactile feedback, navigation, and pushability of the device may make it prone to unintended consequences.
\end{abstract}




\section{Introduction}

ERCP is an important endoscopic therapeutic procedure for a variety of biliary and pancreatic indications. It is estimated that there are 500,000-660,000 ERCPs performed annually in the USA [1]. Infection transmission through ERCPs has been an evolving issue in the last few years. Multiple studies have pointed to infection transmission with reusable duodenoscopes with potential problems related to duodenoscope reprocessing, duodenoscope complex designs, and human factors [1]. The US Food and Drug Administration (FDA) in August 2019 released a safety communication recommending transition to duodenoscopes with innovative designs that facilitate or eliminate the need for reprocessing [2]. Since then, there has been a significant amount of development into disposable duodenoscope components and single-use duodenoscopes [3, 4]. We describe here a case of esophageal perforation after using a single-use disposable duodenoscope (EXALT Model D; Boston Scientific Corporation). To our knowledge, this is the first reported case of an esophageal perforation after the FDA approval of this device in December 2019 [5].

\section{Case Report/Case Presentation}

A 92-year-old male was referred to our hospital after developing an esophageal perforation during ERCP. His past medical history was significant for atrial fibrillation on apixaban, deep-vein thrombosis, and congestive heart failure due to nonischemic cardiomyopathy, hypertension, COPD, mild pulmonary fibrosis, nephrolithiasis, and gout. Three days prior, he had been admitted to an outside community hospital for elevated liver enzymes and fever. He was diagnosed with choledocholithiasis and acute cholangitis and was scheduled for an ERCP. Preoperative antibiotics were started, and the procedure was performed under general anesthesia. A single-use disposable duodenoscope (EXALT Model D; Boston Scientific Corporation) was used for the procedure by an experienced community gastroenterologist with $>2,000$ life-time ERCPs. During scope insertion, gentle force was applied to pass through the gastroesophageal junction, which caused minimal bleeding. To inspect the area, the singleuse duodenoscope was removed, and a reusable gastroscope (GIF-HQ190; Olympus America, Central Valley, PA, USA) was inserted which revealed a $10-\mathrm{mm}$ linear perforation in the esophagus just proximal to the Z-line. To cover the perforation, a fully covered esophageal stent (WallFlex; Boston Scientific Corporation) was deployed. The patient was then transferred to our hospital for further management. Abdominal X-rays showed stent migration below the diaphragm to the stomach (shown in Fig. 1). Hence, a repeat EGD was performed which showed a 10-mm esophageal perforation with purulent fluid just proximal to the Z-line at $41 \mathrm{~cm}$ from the incisors (shown in Fig. 2). The prior esophageal stent which had migrated to the stomach was removed with a pair of rat-toothed forceps. A new esophageal stent (EndoMAXX $23 \mathrm{~mm} \times 150 \mathrm{~mm}$; Merit Medical, Jordan, UT, USA) was deployed at $30 \mathrm{~cm}$ from the incisors covering the defect. Additionally, a stent fixation device (OTSC-Stentfix; Ovesco Endoscopy, Cary, NC, USA) was used to anchor the stent to the esophageal wall at its proximal edge. The patient tolerated the procedure well and was kept nil-per-os for 2 days postoperatively. Oral intake was started on day 3 , and the patient was discharged. Follow-up outpatient endoscopy after 6 weeks revealed the esophageal stent in place at the middle to lower esophagus, bridging the area of the prior perforation. Stent removal was accomplished with a pair of rat-toothed forceps, revealing an 8-mm scar with healthy granulation tissue in the lower third of the esophagus labeled with an arrow (shown in Fig. 3). There was no evidence of fistula formation in the lower esophagus or at the gastroesophageal junction. The patient was able to tolerate a regular diet without any difficulty.

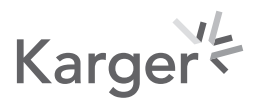


Cheema et al.: Esophageal Perforation after Using a Single-Use Disposable Duodenoscope

Fig. 1. Abdominal X-ray showing the prior esophageal stent that migrated to the stomach.

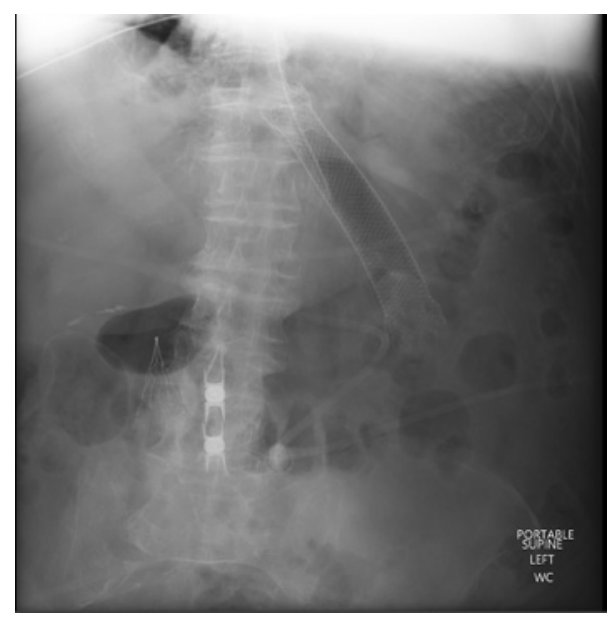

Fig. 2. Esophageal perforation proximal to the gastroesophageal junction.

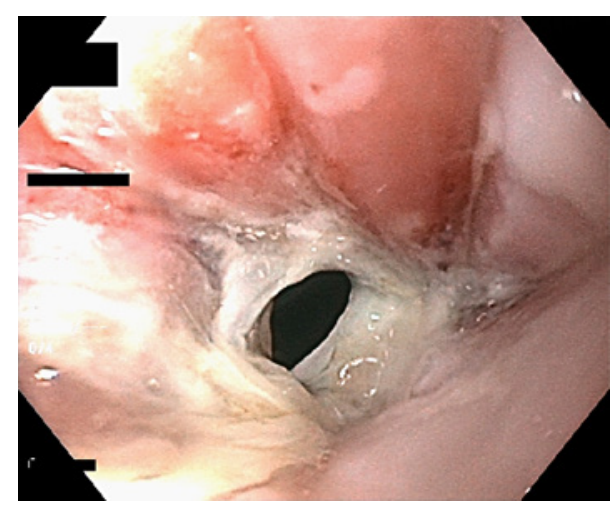

Fig. 3. Recovery after stent removal showing healthy granulation tissue at the site of the perforation labeled with an arrow.

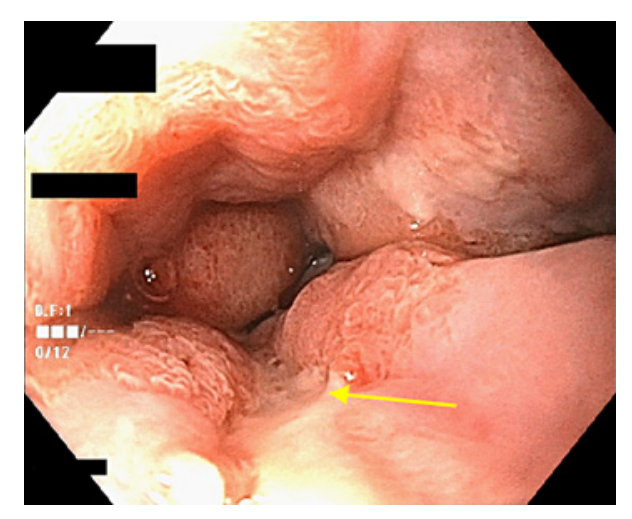

\section{Discussion/Conclusion}

The EXALT Model D single-use duodenoscope (Boston Scientific Corporation) is a relatively newer device with only a few centers in the world with experience in using this device. The device comes in sterile packaging for single use and is recycled through approved thirty party vendors. This device is lighter than the standard reusable duodenoscope, and the tactile feedback, navigation, and pushability are different [3]. A few expert endoscopists at tertiary care academic centers in the USA and Europe have tested this device achieving adequate 
results in biliary cannulation and other common maneuvers. In a case series study in 2020, Muthusamy et al. [6] reported a completion rate of ERCPs of 96.7\% (58/60) using the EXALT Model D single-use duodenoscope and 3.3\% (2/60) using the single-use duodenoscope followed by crossover to a reusable duodenoscope to complete the procedure. The median overall satisfaction score by the endoscopist was $9 / 10$. The 7-day rate of post-procedure serious adverse events was similar to standard ERCP practice.

A single-center randomized controlled trial reported by Bang et al. [7] in 2020 compared performance characteristics of a single-use duodenoscope with a reusable duodenoscope and measured the number of attempts to achieve successful cannulation of the desired duct in 98 patients. There was no significant difference in overall rates of successful cannulation between single-use duodenoscopes (46/48 patients [95.8\%]) versus reusable duodenoscopes (50/50 patients [100\%]) ( $p=0.237)$; however, the median number of attempts to achieve selective ductal access was significantly lower for the single-use cohort ( 2 vs. $5, p=0.013$ ). Other parameters, namely, the ease of duodenoscope passage into the stomach ( $p=0.047)$, image quality $(p<0.001)$, and air-water button functionality $(p<0.001)$ were significantly worse for single-use duodenoscopes.

The medical economics of disposable duodenoscopes can provide some challenges for their widespread adoption. The per-procedure cost of a disposable duodenoscope in the USA has been reported in this study by Bang et al. [8] in 2019. The per-procedure cost of a reusable duodenoscope can vary from USD 1,318 to USD 2,068 for centers performing at the 25th percentile of procedure volume ( $\leq 50$ ERCPs/year) and from USD 797 to USD 1,547 for centers performing at the 75 th percentile of ERCP procedure volume ( $\geq 150$ ERCPs/year), based on infection rates of $0.4 \%-1 \%$, respectively. Using mathematic modeling, the authors estimated that the break-even cost of disposable duodenoscopes in low-volume centers ( $\leq 50$ ERCPs / year) was $\geq$ USD 1,300, depending on infection rates. For large-volume centers ( $\geq 150$ ERCPs/ year), the break-even cost was $\geq$ USD 800 .

Based on hospital contractual agreements with Boston Scientific Corporation, the Exalt Model D single-use duodenoscope is presently available in the USA between USD 1900 and USD 4,000 [9]. It is currently unknown how this additional cost will be covered by insurance. The Centers for Medicare \& Medicaid Services approved a prospective payment formula for single-use endoscopes for outpatients [8]. It remains to be seen how this will eventually affect patient premiums because of the heterogenous hospital-to-insurer contracts in the USA. There are no corresponding payment formulas for inpatient procedures using single-use endoscopes or for other commercial insurance.

Multiple strategies to decrease infection transmission through duodenoscope reprocessing have been evaluated extensively. Enhanced reprocessing strategies like double highlevel disinfection and liquid chemical sterilization were compared in this study by Gromski et al. [10] in 2021. Both double high-level disinfection and liquid chemical sterilization resulted in a low rate of positive cultures for all organisms; however, neither process completely eliminated positive cultures from reprocessed duodenoscopes, highlighting the need for further improvements in reprocessing technologies. Forbes et al. [11] in 2020 reported about a single-center randomized controlled trial comparing bacterial contamination rates and ERCP technical success rates between duodenoscope with disposable end-caps and traditional duodenoscopes. This trial is still in progress with results expected soon, and it may provide additional information regarding infection transmission through duodenoscope disposable end-caps. Other innovative strategies to seal the distal end of duodenoscopes while preserving optics and other endoscope functionalities have also been studied with promising results [12].

Single-use disposable duodenoscopes can help improve or eliminate the rates of exogenous infections; however, endogenous infections which are the predominant cause of 
post-procedure cholangitis may not be decreased, necessitating the continual use of standard infection control measures and practicing safe procedural techniques ensuring adequate drainage [7]. Bilal et al. [13] reported the possibility of using the single-use duodenoscopes in patients with COVID-19. Bang et al. [7] suggested the possibility of using the single-use duodenoscope in high-risk patients with cholangiocarcinoma and biliary stents, immunocompromized patients, or in known carriers of multidrug-resistant organisms.

Complications with ERCPs, especially for therapeutic indications, are higher than general diagnostic gastrointestinal procedures. Christensen et al. [14] in 2004 reported that the 30-day complication rate with ERCPs was 15.9\%; the procedure-related mortality rate was $1.0 \%$, cholangitis $5.0 \%$, pancreatitis $3.8 \%$, cardiorespiratory complications $2.3 \%$, perforation $1.1 \%$, and hemorrhage in $0.9 \%$ of procedures. However, Merchea et al. [15] reported that the overall incidence of perforation for esophagogastroduodenoscopy was $0.033 \%(72 / 217,507)$. These studies illustrate that perforations although present are relatively rare.

Based on discussions with the community gastroenterologist who performed the ERCP in this case, we feel that this perforation likely occurred because the single-use duodenoscope tip bent inadvertently at the lower esophageal sphincter and due to a subsequent miscalculation of the amount of force needed to traverse the gastroesophageal junction with the single-use duodenoscope.

We felt the need to report this case because without sufficient training and establishing whether there is a learning curve, the differences in tactile feedback, navigation, and pushability of the single-use duodenoscope can make it prone to unintended consequences increasing patient morbidity and mortality, eventually affecting cost-effectiveness of this device. These costs must be balanced with reusable duodenoscope reprocessing and labor costs to get an accurate comparison. Other considerations include image quality, environmental impact on recycling, and the use of single-use duodenoscopes outside of the expert tertiary level centers need to be evaluated. Further large-scale multicenter prospective studies are needed to better address some of these concerns.

\section{Acknowledgment}

This manuscript does not include any nonauthor contributors to acknowledge.

\section{Statement of Ethics}

Written informed consent was obtained from the patient for publication of this case report and any accompanying images. This study protocol was reviewed, and the need for approval was waived by the Ethics committee of UF College of Medicine - Jacksonville.

\section{Conflict of Interest Statement}

The authors of this manuscript do not have any conflict of interest to declare.

\section{Funding Sources}

This manuscript did not receive any funding.

\section{Karger'}


Cheema et al.: Esophageal Perforation after Using a Single-Use Disposable

Duodenoscope

\section{Author Contributions}

Bakht S. Cheema (B.S.C.) reported the concept and design, acquisition of data, drafting of manuscript, and critical revision of manuscript. Maged Ghali (M.G.) reported the concept and design, drafting of manuscript, and critical revision of manuscript. Ron Schey (R.S.) reported the concept and design, drafting of manuscript, and critical revision of manuscript. Ziad Awad (Z.A.) reported the concept and design and acquisition of data. Bruno Ribeiro (B.R.) reported concept and design, acquisition of data, drafting of manuscript, and critical revision of manuscript. All the authors (B.S.C., M.G., R.S., Z.A., and B.R.) approved the final version of the manuscript.

\section{Data Availability Statement}

All data generated or analyzed during this study are included in this article. Further inquiries can be directed to the corresponding author.

\section{References}

1 Rahman MR, Perisetti A, Coman R, Bansal P, Chhabra R, Goyal H. Duodenoscope associated infections: update on an emerging problem. Dig Dis Sci. 2019;64:1409-18.

2 US Food and Drug Administration. FDA recommends health care facilities and manufacturers begin transitioning to duodenoscopes with disposable components to reduce risk of patient infection. 2019 Aug 29. Available from: https://www.fda.gov/news-events/press-announcements/fda-recommends-health-carefacilities-andmanufacturers-begin-transitioning-duodenoscopes-disposable Accessed $2021 \mathrm{Apr} 7$.

3 Ross AS, Bruno MJ, Kozarek RA, Petersen BT, Pleskow DK, Sejpal DV, et al. Novel single-use duodenoscope compared with 3 models of reusable duodenoscopes for ERCP: a randomized bench-model comparison. Gastrointest Endosc. 2020;91:396-403.

4 Trindade AJ, Copland A, Bhatt A, Bucobo JC, Chandrasekhara V, Krishnan K, et al. Single-use duodenoscopes and duodenoscopes with disposable end caps. Gastrointest Endosc. 2021;93(5):997-1005.

5 US Food and Drug Administration. FDA clears first fully disposable duodenoscope, eliminating the potential for infections caused by ineffective reprocessing. 2019. Available from: https://www.fda.gov/news-events/ press-announcements/fda-clears-first-fully-disposable-duodenoscope-eliminating-potential-infectionscaused-ineffective Accessed 2021 Apr 7.

6 Muthusamy VR, Bruno MJ, Kozarek RA, Petersen BT, Pleskow DK, Sejpal DV, et al. Clinical evaluation of a single-use duodenoscope for endoscopic retrograde cholangiopancreatography. Clin Gastroenterol Hepatol. 2020;18:2108-17.

7 Bang JY, Hawes R, Varadarajulu S. Equivalent performance of single-use and reusable duodenoscopes in a randomised trial. Gut. 2021;70(5):838-44.

8 Bang JY, Sutton B, Hawes R, Varadarajulu S. Concept of disposable duodenoscope: at what cost? Gut. 2019;68: 1915-7.

9 Holzwanger EA, Bilal M, Saperia J, Cohen J, Sawhney MS, Berzin TM, et al. Duodenoscope-related infections and potential role of single-use duodenoscopes. VideoGIE. 2020;5:628-9.

10 Gromski MA, Sieber MS, Sherman S, Rex DK. Double high-level disinfection versus liquid chemical sterilization for reprocessing of duodenoscopes used for ERCP: a prospective, randomized study. Gastrointest Endosc. 2021;93:927-31.

11 Forbes N, Elmunzer BJ, Allain T, Chau M, Koury HF, Bass S, et al. Infection control in ERCP using a duodenoscope with a disposable cap (ICECAP): rationale for and design of a randomized controlled trial. BMC Gastroenterol. 2020 Mar;20(1):64.

12 Pasricha PJ, Miller S, Carter F, Humphries R. Novel and effective disposable device that provides 2-way protection to the duodenoscope from microbial contamination. Gastrointest Endosc. 2020;92:199-208.

13 Bilal M, Berzin TM, Cohen J, Sawhney MS, Pleskow DK. ERCP in patients with COVID-19 infection: is a singleuse duodenoscope the safer option? Endoscopy. 2020;52(10):932.

14 Christensen M, Matzen P, Schulze S, Rosenberg J. Complications of ERCP: a prospective study. Gastrointest Endosc. 2004;60:721-31.

15 Merchea A, Cullinane DC, Sawyer MD, Iqbal CW, Baron TH, Wigle D, et al. Esophagogastroduodenoscopy-associated gastrointestinal perforations: a single-center experience. Surgery. 2010;148:876-80; discussion 881-2. 